\title{
DATA QUALITY EVALUATION AND APPLICATION POTENTIAL ANALYSIS OF TIANGONG-2 WIDE-BAND IMAGING SPECTROMETER
}

\author{
Bangyong Qin, Leijuan Li, Shengyang Li \\ Key Laboratory of Space Utilization, Technology and Engineering Center for Space Utilization, Chinese Academy of Sciences, \\ Beijing 100094, P. R. China - (qinby, lileijuan, shyli)@csu.ac.cn
}

KEY WORDS: Tiangong-2, Wide-band Imaging Spectrometer, Remote sensing, Data quality, Quality evaluation

\begin{abstract}
:
Tiangong-2 is the first space laboratory in China, which launched in September 15, 2016. Wide-band Imaging Spectrometer is a medium resolution multispectral imager on Tiangong-2. In this paper, the authors introduced the indexes and parameters of Wideband Imaging Spectrometer, and made an objective evaluation about the data quality of Wide-band Imaging Spectrometer in radiation quality, image sharpness and information content, and compared the data quality evaluation results with that of Landsat-8. Although the data quality of Wide-band Imager Spectrometer has a certain disparity with Landsat-8 OLI data in terms of signal to noise ratio, clarity and entropy. Compared with OLI, Wide-band Imager Spectrometer has more bands, narrower bandwidth and wider swath, which make it a useful remote sensing data source in classification and identification of large and medium scale ground objects. In the future, Wide-band Imaging Spectrometer data will be widely applied in land cover classification, ecological environment assessment, marine and coastal zone monitoring, crop identification and classification, and other related areas.
\end{abstract}

\section{INTRODUCTION}

On orbit data quality evaluation of remote sensing payload is a vital part in data processing and data quality control of satellite ground system(Chen et al., 2004). Many operational satellites carry out data quality analysis and evaluation regularly, to grasp the change of data quality, and improve and control the quality of data products through orbit calibration (Brian et al., 2015, Ron, 2015, Hong, 2010, Zhang et al., 2010, Zhang, 2009, Wang and Tian, 2007).

Tiangong-2 is the first true space laboratory of China, which developed and launched on September 15, 2016 by China Manned Space Project, to carry out space science and application experiment (Qin et al, 2017). Wide-band Imaging Spectrometer (MWI) is a mid-resolution multispectral imager on Tiangong-2 (Qin et al., 2017). It will mainly used for remote sensing monitoring and application in ocean, land and atmosphere.

At present, MWI has acquired abundant image data of earth observation. In order to verify its imaging quality, the quality of the image data was objectively evaluated by image quality evaluation indexs, and the evaluation results were compared with the OLI data of Landsat-8. In addition, the application potential and application direction of MWI data were also discussed.

\section{DATA}

MWI of Tiangong-2 uses push-broom imaging model and with an observation swath of $300 \mathrm{~km}$, its spectrum range cover visible near-infrared (VNI), short-wave infrared (SWI) and thermal infrared (INF). The camera parameters are shown in Table 1 (Qin et al, 2017).

\begin{tabular}{|l|c|c|c|}
\hline $\begin{array}{l}\text { Spectral } \\
\text { segments }\end{array}$ & Spectral range & $\begin{array}{c}\text { Band } \\
\text { numbers }\end{array}$ & $\begin{array}{c}\text { Spatial } \\
\text { resolution }\end{array}$ \\
\hline VNI & $0.4 \mu \mathrm{m}-1.0 \mu \mathrm{m}$ & 14 & $100 \mathrm{~m}$ \\
SWI & $1 \mu \mathrm{m}-1.7 \mu \mathrm{m}$ & 2 & $200 \mathrm{~m}$ \\
INF & $8 \mu \mathrm{m}-10 \mu \mathrm{m}$ & 2 & $400 \mathrm{~m}$ \\
\hline
\end{tabular}

Table 1. Part of the parameters of MWI

Landsat- 8 is a famous land observation satellite launched by National Aeronautics and Space Administration of America on February 11, 2013 (U.S. Geological Survey, 2015). There are two main loads on the Landsat-8, they are Operational Land Imager (OLI) and Thermal Infrared Sensor (TIRS).

MWI and OLI are both multi-spectral imagers, the spatial resolution of OLI is $30 \mathrm{~m}$, which is higher than that of MWI (100m for VNI, $200 \mathrm{~m}$ for SWI, $400 \mathrm{~m}$ for INF). MWI has more effective bands and narrower spectral bandwidth. The details of the two imagers are shown in Table 2 (U.S. Geological Survey, 2015).

\begin{tabular}{|c|c|c|c|}
\hline \multicolumn{2}{|c|}{ MWI of Tiangong-2 } & \multicolumn{2}{c|}{ OLI of Landsat- 8} \\
\hline $\begin{array}{c}\text { Band } \\
\text { number }\end{array}$ & $\begin{array}{c}\text { Spectrum range } \\
(\mu \mathrm{m})\end{array}$ & $\begin{array}{c}\text { Band } \\
\text { number }\end{array}$ & $\begin{array}{c}\text { Spectrum range } \\
(\mu \mathrm{m})\end{array}$ \\
\hline VNI-b1 & $0.403 \sim 0.423$ & & \\
VNI-b2 & $0.433 \sim 0.453$ & OLI-b1 & $0.433-0.453$ \\
VNI-b3 & $0.480 \sim 0.500$ & OLI-b2 & $0.450-0.515$ \\
VNI-b4 & $0.510 \sim 0.530$ & & \\
VNI-b5 & $0.555 \sim 0.575$ & OLI-b3 & $0.525-0.6$ \\
VNI-b6 & $0.610 \sim 0.630$ & & \\
VNI-b7 & $0.655 \sim 0.675$ & OLI-b4 & $0.63-0.68$ \\
& & OLI-b8 & $0.5-0.68$ \\
VNI-b8 & $0.6775 \sim 0.6875$ & & \\
VNI-b9 & $0.740 \sim 0.760$ & & \\
VNI-b10 & $0.810 \sim 0.830$ & & \\
VNI-b11 & $0.845 \sim 0.885$ & OLI-b5 & $0.845-0.885$ \\
VNI-b12 & $0.895 \sim 0.915$ & & \\
VNI-b13 & $0.930 \sim 0.950$ & & \\
VNI-b14 & $0.970 \sim 0.990$ & &
\end{tabular}




\begin{tabular}{|c|c|c|c|}
\cline { 1 - 1 } SWI-b1 & $1.23 \sim 1.25$ & & \\
& & OLI-b9 & $1.36-1.39$ \\
SWI-b2 & $1.63 \sim 1.65$ & OLI-b6 & $1.56-1.66$ \\
& & OLI-b7 & $2.1-2.3$ \\
INF-b1 & $8.125 \sim 8.825$ & & \\
INF-b2 & $8.925 \sim 9.275$ & & \\
\hline
\end{tabular}

Table 2. Band parameter comparison between MWI and OLI

As shown in table 2, there are several similar bands between MWI and OLI. For MWI, the similar bands are band2, band3, band5, band7, band11 of VNI and band2 of SWI. For OLI, the similar bands are form band1 to band6.

In this paper, two sets of remote sensing images of MWI are selected as test images. Both of them are after radiation corrected and geometrically corrected. The ground cover types of the two image are respectively Dunhuang Desert (Figure 1) and Qinghai lake (Figure 2). The radiation of them are both relatively uniform. Dunhuang Desert is a well-known radiation calibration site in China. Qinghai Lake is the largest inland lake in China.

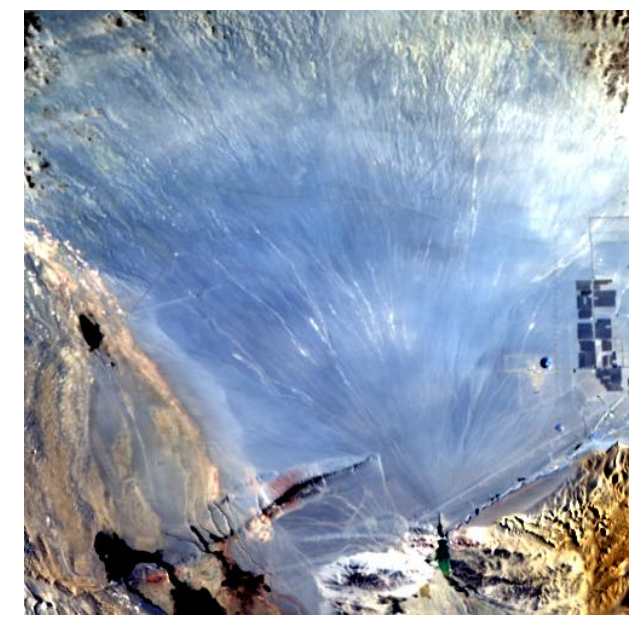

Figure 1. MWI image of Dunhuang Desert (Colour synthesis with band 7 , band 5 and band 3 )

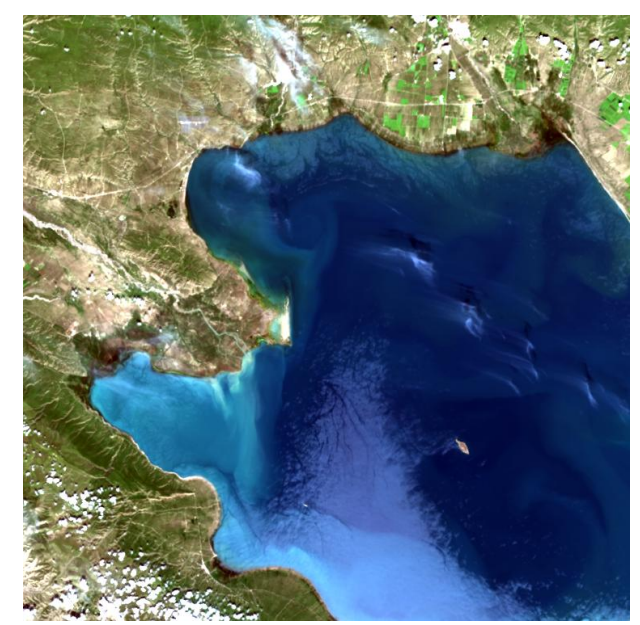

Figure 2. MWI image of Qinghai Lake (Colour synthesis with band 7 , band 5 and band 3 )

In order to compare the data quality with MWI, two images of OLI were taken as comparative data. They were acquired by
OLI in July 2017. Figure 3 is the image of Dunhuang Desert, and figure 4 is the image of Qinghai Lake.

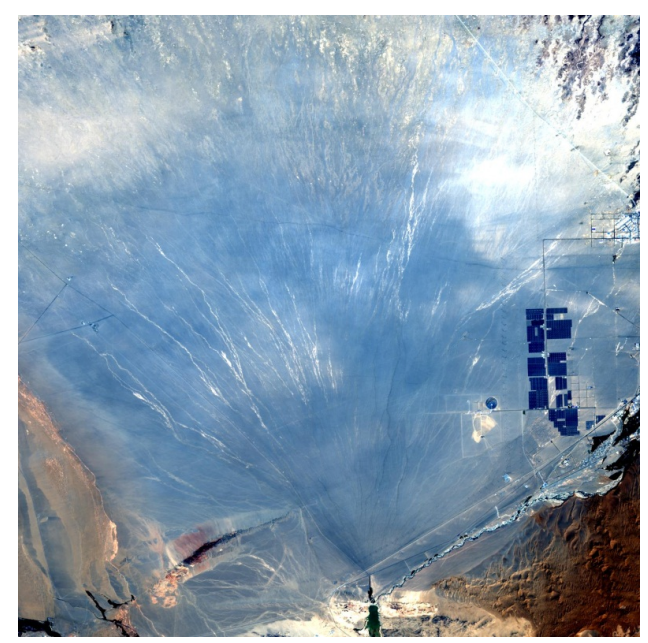

Figure 3. Landsat-8 OLI image of Dunhuang Desert (Colour synthesis with band 4 , band 3 and band 2)

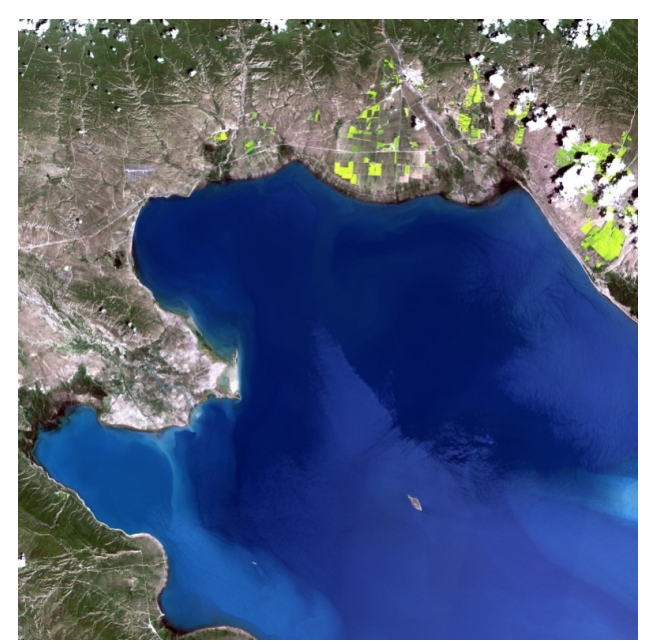

Figure 4. Landsat-8 OLI data in Qinghai Lake (Colour synthesis with band 4 , band 3 and band 2)

\section{METHOD}

In order to comprehensively evaluated the data quality of Tiangong-2 MWI, three types of evaluation elements are adopted in this paper. They are radiation quality, image sharpness and information content. The radiation quality is evaluated with image signal-to-noise ratio (SNR), image contrast and radiation uniform. Image sharpness is evaluated with image clarity index, and information content is evaluated with information entropy.

\subsection{Image signal-to-noise ratio}

Image SNR is the most commonly used index to evaluate the noise in images (Gao, 2008, Corner et al., 2003). In this paper, the image noise estimate method based on region segmentation and residuals statistical is used to calculate the SNR of the test images (Qin et al., 2014, Gao et al., 2007, Gao, 1993). This method is less affected by different feature types and mixed pixel, can get more accurate assessment result (Qin et al., 2014). The calculation equation of image SNR is as follows: 


$$
S N R=20 \lg \left(\frac{M}{\sigma}\right)
$$

where $\quad S N R=$ image signal-to-noise ratio

$M=$ image signal mean

$\sigma=$ image noise standard deviation

\subsection{Image contrast}

Image contrast is often used to reflect the gray contrast and the detail identification degree in images. In this paper, image contrast is measured based on the Gray Level Co-occurrence Matrix (GLCM) (Haralick et al., 1973, Guo and Song, 2005) of image, which can get evaluation results with consistency and comparability, especially for the images with different response range. The calculation equation of image contrast is as follows (Bu, 2012):

$$
C O N=\sum_{i=0}^{L-1} \sum_{j=0}^{L-1}(i-j)^{2} p(i, j)
$$

where $C O N=$ image contrast

$i, j=$ image gray value after normalized

$L=$ the gray levels of normalized image

$p(i, j)=$ the GLCM element of the image

\subsection{Radiation uniform}

Radiation uniform is mainly used for evaluating the relative radiometric correction accuracy of images (Krause, 2004). There are several methods for radiation uniform evaluation, in this paper, the average line standard deviation method is adopted (Hu and zhang, 2008). The calculation equation of image radiation uniform is as follows:

$$
\overline{D N}_{j}=\sqrt{\frac{\sum_{i=1}^{m} D N_{i, j}}{m}} \times 100 \%
$$

where $\quad \overline{D N}_{j}=$ the mean of image gray in column $j$

$i, j=$ row number and column number in image $m=$ total row number of image

$D N_{i, j}=$ the gray value of pixel $(i, j)$ in image

$$
R A=\frac{\sqrt{\left(\sum_{j=1}^{n}\left(\overline{D N_{j}}-\overline{D N}\right)^{2}\right) / n}}{\overline{D N}} \times 100 \%
$$

where $\quad R A=$ image radiation uniform

$j=$ column number in image

$n=$ total column number of image

$\overline{D N}_{j}=$ the mean of image gray in column $j$

$\overline{D N}=$ the mean of image gray

\subsection{Image clarity}

Image clarity is a commonly used quality evaluation index to measure the fuzzy distortion degree of images (Wang et al., 2004). In this paper, a clarity evaluation method based on edge gradient is adopted, which can reflect the sharpness degree of feature edge in the image, and has a high correlation with the modulation transfer function (MTF) of images (Qin et al., 2015,
Zhang and Zhang 2002, Schowengerdt et al., 1996). The calculation equation of image clarity is as follows:

$$
E(i)=\frac{\sum_{k=1}^{L} g_{i}(k)^{2}}{\left(\sum_{k=1}^{L} g_{i}(k)\right)^{2}}
$$

where $\quad E(i)=$ sharpness value at edge point $i$

$i=$ number of edge point

$k=$ the subscript of the gradient vector

$L=$ length of gradient vector

$g_{i}(k)=$ the $k$ element of the gradient vector at edge

point $i$

$$
C L A=\frac{\sum_{i=1}^{N} E(i)}{N}
$$

where $\quad C L A=$ image clarity

$i=$ number of edge point

$N=$ the total numbers of edge points

$E(i)=$ sharpness value at edge point $i$

\subsection{Information entropy}

Information entropy can reflect the richness of image information and usually be used as a reference factor during image quality evaluation. In this paper, Shannon entropy is selected to evaluate the information entropy of the image (Zhou and Tian, 2008). The calculation equation of image information entropy is as follows:

$$
E N T=-\sum_{i=\min }^{\max } p_{i} \log _{2} p_{i}
$$

where $\quad E N T=$ information entropy of image

$i=$ gray value of image pixel in an image

$\min , \max =$ the minimum and maximum of gray value equal $i$

$p_{i}=$ pixel probability in an image which gray value

\section{RESULTS AND ANALYSIS}

\subsection{Quality evaluation results of MWI}

The image quality evaluation results of Tiangong-2 MWI data are as follows:

\begin{tabular}{|c|c|c|c|c|c|}
\hline band & SNR & contrast & $\begin{array}{c}\text { radiation } \\
\text { uniform }\end{array}$ & clarity & entropy \\
\hline 1 & 57.1 & 0.6 & 0.2 & 74.8 & 6.7 \\
2 & 55.0 & 0.6 & 0.5 & 73.4 & 6.3 \\
3 & 53.1 & 0.8 & 0.9 & 74.0 & 7.4 \\
4 & 52.1 & 1.0 & 1.2 & 74.5 & 7.4 \\
5 & 50.2 & 1.3 & 1.5 & 75.3 & 7.4 \\
6 & 48.4 & 1.5 & 1.8 & 75.6 & 7.5 \\
7 & 47.6 & 1.5 & 1.9 & 75.7 & 7.7 \\
8 & 47.2 & 1.6 & 1.9 & 75.8 & 7.7 \\
9 & 47.5 & 1.8 & 1.9 & 80.1 & 7.6 \\
10 & 46.6 & 1.7 & 2.5 & 80.0 & 7.1 \\
11 & 46.6 & 1.7 & 2.3 & 80.2 & 7.4 \\
12 & 45.9 & 1.6 & 2.5 & 80.4 & 6.6
\end{tabular}




\begin{tabular}{|c|c|c|c|c|c|}
\hline 13 & 42.4 & 1.2 & 2.4 & 80.8 & 4.8 \\
14 & 44.7 & 1.6 & 2.2 & 80.3 & 6.6 \\
15 & 40.1 & 1.1 & 2.3 & 80.0 & 5.8 \\
16 & 39.3 & 1.1 & 2.3 & 78.1 & 5.4 \\
17 & 63.9 & 0.6 & 0.1 & 81.8 & 7.6 \\
18 & 61.5 & 0.5 & 0.1 & 78.2 & 8.3 \\
Mean & 49.4 & 1.2 & 1.6 & 77.7 & 7.0 \\
Std & 6.6 & 0.4 & 0.8 & 2.7 & 0.9 \\
\hline
\end{tabular}

Table 3. Quality evaluation results of MWI data in Dunhuang Desert

\begin{tabular}{|c|c|c|c|c|c|}
\hline band & SNR & contrast & $\begin{array}{c}\text { radiation } \\
\text { uniform }\end{array}$ & clarity & entropy \\
\hline 1 & 51.7 & 0.9 & 4.3 & 73.5 & 8.9 \\
2 & 47.0 & 0.9 & 5.6 & 72.7 & 8.4 \\
3 & 46.0 & 1.0 & 6.9 & 72.5 & 9.5 \\
4 & 45.6 & 1.0 & 7.7 & 72.2 & 9.5 \\
5 & 42.6 & 0.7 & 9.5 & 72.4 & 10.0 \\
6 & 37.6 & 0.6 & 18.5 & 72.4 & 10.0 \\
7 & 34.6 & 0.7 & 24.6 & 72.1 & 9.9 \\
8 & 34.1 & 0.7 & 26.2 & 72.7 & 9.9 \\
9 & 36.2 & 0.3 & 30.0 & 68.2 & 10.3 \\
10 & 35.8 & 0.3 & 32.5 & 68.4 & 10.0 \\
11 & 35.7 & 0.3 & 35.1 & 67.9 & 10.0 \\
12 & 35.8 & 0.3 & 33.6 & 68.7 & 9.6 \\
13 & 36.3 & 0.3 & 26.4 & 67.4 & 8.9 \\
14 & 36.7 & 0.3 & 29.0 & 68.0 & 9.5 \\
15 & 40.1 & 0.3 & 14.6 & 65.2 & 9.6 \\
16 & 36.2 & 0.4 & 24.5 & 68.1 & 8.4 \\
17 & 55.2 & 0.7 & 1.3 & 64.4 & 9.5 \\
18 & 56.2 & 0.7 & 1.0 & 64.7 & 9.6 \\
Mean & 41.3 & 0.6 & 18.4 & 69.5 & 9.5 \\
Std & 7.1 & 0.3 & 11.7 & 3.0 & 0.5 \\
\hline
\end{tabular}

Table 4. Quality evaluation results of MWI data in Qinghai Lake

In table 3 and table 4, the Mean is the average value of all the bands, and the Std is the standard deviation of all the bands.

As shown in table 3 and table 4 , the evaluation results of the two images are different in some degree. Radiation uniform and contrast have strong correlation with the feature types in images, the other indexes, SNR, clarity and entropy, are less affected by the feature types, the evaluation results of which can reflect the overall quality of image data.

Except the index of radiation uniform, the evaluation results of other indexes have good consistency during different bands of the same image, which indicates that the image quality of each bands of MWI are relatively balanced.

The average SNR of the two images are both more than $40 \mathrm{~dB}$, and the average clarity of the two images is no less than 70, data products quality can meet the application requirements.

\subsection{Comparison of data quality and application potential analysis}

To further illustrate the data quality of the MWI, the quality evaluation results of MWI were compared with those of OLI in the similar bands, the results were as follow:

\begin{tabular}{|c|c|c|c|c|c|}
\hline & SNR & $\begin{array}{c}\text { cont } \\
\text { rast }\end{array}$ & $\begin{array}{c}\text { radiation } \\
\text { uniform }\end{array}$ & clarity & entropy \\
Mean(MWI) & 48.6 & 1.2 & 1.6 & 76.1 & 6.9 \\
Std(MWI) & 5.1 & 0.4 & 0.7 & 2.4 & 0.8 \\
Mean(OLI) & 53.9 & 0.8 & 0.8 & 85.4 & 11.5 \\
Std(OLI) & 2.8 & 0.3 & 0.5 & 0.5 & 0.3 \\
\hline
\end{tabular}

Table 5. Data quality evaluation results comparison between MWI and OLI in image of Dunhuang Desert

\begin{tabular}{|c|c|c|c|c|c|}
\hline & SNR & $\begin{array}{c}\text { cont } \\
\text { rast }\end{array}$ & $\begin{array}{c}\text { radiation } \\
\text { uniform }\end{array}$ & clarity & entropy \\
Mean(MWI) & 40.4 & 0.7 & 17.7 & 71.0 & 9.4 \\
Std(MWI) & 5.0 & 0.2 & 11.0 & 2.1 & 0.7 \\
Mean(OLI) & 52 & 0.6 & 2.5 & 84.8 & 11.3 \\
Std(OLI) & 3.6 & 0.2 & 0.4 & 1.1 & 0.3 \\
\hline
\end{tabular}

Table 6. Data quality evaluation results comparison between MWI and OLI in image of Qinghai Lake

In table 5 and table 6, the Mean and Std were the average value and the standard deviation of the similar bands. For MWI, the similar bands are band2, band3, band5, band7, band11 and band16. For OLI, the similar bands are form band1 to band6.

As shown in table 5 and table 6 , the image contrast of MWI data is larger than that of OLI in both Qinghai Lake and Dunhuang Desert. In other indexes, such as SNR, clarity, entropy and radiation uniform, the data quality of MWI still has a gap compared with that of OLI.

The spatial resolution of OLI data is higher than that of MWI, thus it can present more details of ground features. While MWI has more bands, narrower bandwidth and wider swath, it can provide more spectral information of the ground objects. In the classification and recognition application of large or medium scale ground objects, it will be an effective method to combined use these two data sources.

At present, the MWI data product has been applied in many directions, such as land cover classification, coastal monitoring, lake monitoring, ecological environment evaluation, crop classification and so on, and have shown its great application potential (Liu, et al., 2017, Technology and Engineering Center for Space Utilization, Chinese Academy of Sciences, 2018).

\section{CONCLUSIONS}

The radiation quality, image sharpness and information content of Tiangong-2 MWI data are well and can meet the application requirements, although the data quality of MWI still has a gap compared with that of OLI in the overall.

There are several similar bands between MWI and OLI, researchers can combined use these two data sources in remote sensing applications.

Since the band number of MWI is more than that of OLI, it has more advantages in classification and identification of large and medium scale ground objects.

Tiangong-2 MWI data has broad application prospects, and will be widely used in land cover classification, ecological environment assessment, marine and coastal zone monitoring, crop identification and classification, and other related fields. 


\section{ACKNOWLEDGEMENTS}

Thank China Manned Space Engineering for providing WideBand Imaging Spectrometer data. The images used in this paper were downloaded from the website: http://www.msadc.cn.

\section{REFERENCES}

Brian, M., James, S., Ron, M., Prasad, S. T., 2015. Landsat-8 Sensor Characterization and Calibration. Remote Sensing, (3), pp. 2279-2282. https://doi.org/10.3390/rs70302279.

Bu, Y. D., 2012. Research on image texture feature extraction. Shandong Normal University, Jinan, China.

Chen, Z. C.,2004. A Research on the Technology of On-orbit Calibration and Validation of China DMC Microsatellite. Institute of remote sensing applications of the Chinese Academy of Sciences, Beijing, China.

Corner, B., Narayanan, R., Reichenbach, S., 2003. Noise estimation in remote sensing imagery using data masking. International Journal of Remote Sensing, 24(4), pp. 689-702.

Gao, B. C.,1993. An operational method for estimating signal to noise ratios from data acquired with imaging spectrometers. Remote Sensing of Environment, 43(1), pp. 23-33.

Gao, L. R., Zhang, B., Zhang, X., Sheng, Q., 2007. Study on the Method for Estimating the Noise in Remote Sensing Images Based on Local Standard Deviations. Journal of Reomote Sensing, (2), pp. 201-208.

Gao, L. R., Zhang, B., Zhang, X., Zhang, W., Tong, Q.,2008. A new operational method for estimating noise in hyperspectral images. IEEE Geoscience and Remote Sensing Letters, 5(1), pp. 83-87.

Guo, D. J., Song, Z. C., 2005. A Study on Texture Image Classifying Based on Gray-level Co-occurrence Matrix. Forestry Machinery \& Woodworking Equipment, 33(7), pp. 2123.

Haralick, R. M., Shanmugam, K., Dinstein, I. H., 1973. Textural features for image classification. IEEE Transactions on Systems, Man and Cybernetics, (6), pp. 610-621.

Hong, Z. G., Cong, N., Tang, X. M., Li, C. H., 2010. The Elementary Study on the Geometric Correction Assessment of CBERS-02B Data. Geomatics \& Spatial Information Technology, 33(4), pp. 31-34.

U.S. Geological Survey, Landsat 8 OLI (Operational Land Imager) and TIRS (Thermal Infrared Sensor), https://lta.cr.usgs.gov/L8 (1 January 2015)

Technology and Engineering Center for Space Utilization, Chinese Academy of Sciences, Data Application, http://www.msadc.cn/en/cgzs/sjyy/ (1 February 2018)

Hu, Y. F., Zhang, Y. F., 2008. Analysis of Relative Radiometric Calibration Accuracy of Space Camera. Spacecraft Recovery \& Remote Sensing, 28(4), pp. 54-57.

Krause, K. S., 2004. Relative radiometric characterization and performance of the QuickBird high-resolution commercial imaging satellite. In: the SPIE 49th Annual Meeting, International Society for Optics and Photonics, Optical Science and Technology, pp. 35-44.

Liu, K., Qin, B. Y., Mu, L. L., Li, S. Y., 2017. Dynamic monitoring and change analysis of Reclamation Based on multi temporal remote sensing images -- a case study of some coastal counties and cities in Liaoning Province. Marine Environmental Science, 36(6), pp. 943-949.

Qin, B. Y., Hong, B., Zhang, Z., Yang, X. F., Li, Z. W., 2014. A generally applicable noise-estimating method for remote sensing images. Remote Sensing Letters, 5(5), pp. 481-490. https://doi.org/10.1080/2150704X.2014.923126

Qin, B. Y., Li, S. Y., Hao, Z. W., Liu, K., Wang, B., 2017. Earth observation data of the Tiangong-2 Space Laboratory (2016-2017). China Scientific Data, 2(4).

Qin, B. Y., Shang, R., Li, S. Y., Hei, B. Q., Liu, Z. W., 2015. Reliable clarity automatic-evaluation method for optical remote sensing images. In: Applied Optics and Photonics China (AOPC 2015), Image Processing and Analysis. Beijing, China, May 5-7. https://doi.org/10.1117/12.2202971

Ron, M., Julia, B., Raviv, L., Brian, M., Esad, M., Lawrence, O., Pat, S., Kelly, V., James, S., Richard, M., Prasad S. T., 2015. Landsat-8 Operational Land Imager (OLI) Radiometric Performance On-Orbit. Remote Sensing, (2), pp. 2208-2237. DOI:10.3390/rs70202208.

Schowengerdt, R. A., Basedow, R. W., Colwell, J. E.,1996. Measurement of the HYDICE system MTF from flight imagery. In: SPIE Proceedings, Hyperspectral Remote Sensing and Applications, (2821), pp. 127-136.

Wang, H. N., Zhong, W., Wang, J., Xia, D. S., 2004. Research of Measurement for Digital Image Definition. Journal of Image and Graphics, 9(7), pp. 828-831.

Wang, Q. J., Tian, Q. J.,2007. Quality Evaluation of LISS3 Image from IRS - P6 Satellite. Geography and Geo-information Science, 23(3), pp. 11-14.

Zhang, L. F., 2009. Preliminary evaluation of HJ-1A satellite hyperspectral remote sensing image quality. In: The Seventh Conference on imaging spectroscopy technology and applications, Gui Lin, China, pp. 39-44.

Zhang, X., Zhang, B., 2002. Image Quality Assessment for the Infrared Multi-Spectral Scanner of the Chinese-Brazil Earth Resources Satellite. Journal of Image and Graphics, 7(6), pp. 581-586.

Zhang, X. H., Zhang, L. F., Wang, J. N., Tong, Q. X., 2010. Comprehensive evaluation of HJ-1A satellite hyperspectral remote sensing image quality. In: The Eighth Conference on imaging spectroscopy technology and applications, and interdisciplinary forum.

Zhou, Y. J., Tian, Q. J., 2008. Image Quality Evaluation of EO1 Hyperion Sensor. Geo-information Sience, (5), pp. 678-683. 